\title{
Rendering of Pressure and Textures Using Wearable Haptics in Immersive VR Environments*
}

\author{
Giovanni Spagnoletti ${ }^{\dagger}$ \\ University of Siena, Italy
}

\author{
Leonardo Meli \\ University of Siena, Italy
}

Claudio Pacchierotti

CNRS, Univ Rennes, Inria, IRISA, France

\author{
Tommaso Lisini Baldi \\ University of Siena, Italy
}

\author{
Guido Gioioso \\ University of Siena, Italy
}

Domenico Prattichizzo

University of Siena, Italy

Istituto Italiano di Tecnologia, Italy

\begin{abstract}
Haptic systems have only recently started to be designed with wearability in mind. Compact, unobtrusive, inexpensive, easy-to-wear, and lightweight haptic devices enable researchers to provide compelling touch sensations to multiple parts of the body, significantly increasing the applicability of haptics in many fields, such as robotics, rehabilitation, gaming, and immersive systems. In this respect, wearable haptics has a great potential in the fields of virtual and augmented reality. Being able to touch virtual objects in a wearable and unobtrusive way may indeed open new exciting avenues for the fields of haptics and VR. This work presents a novel wearable haptic system for immersive virtual reality experiences. It conveys the sensation of touching objects made of different materials, rendering pressure and texture stimuli through a moving platform and a vibrotactile abbrv-doi-hyperref-narrowmotor. The device is composed of two platforms: one placed on the nail side of the finger and one in contact with the finger pad, connected by three cables. One small servomotor controls the length of the cables, moving the platform towards or away from the fingertip. One voice coil actuator, embedded in the platform, provides vibrotactile stimuli to the user.
\end{abstract}

Index Terms: Hardware-Tactile and hand-based interfacesHaptic devices Human-centered computing-Human computer interaction (HCI) - Interaction paradigms - Virtual reality;

\section{INTRODUCTION}

Wearable haptics is recently attracting great attention in the fields of robotics, haptics, and mechanical engineering. Being able to provide effective haptic stimuli through lightweight, inexpensive, and unobtrusive devices can significantly enlarge the applications of haptic systems to new and exciting fields, such as gaming, rehabilitation, and remote maintenance. Recently, we have also seen a few start-up companies taking up this challenge and start developing wearable haptic devices for the fingertips. For example, Tactai (USA) has presented a fingertip wearable haptic device able to render pressure, texture, and the sensation of making and breaking contact with virtual objects $[4,6]$. It can apply up to $6 \mathrm{~N}$ to the fingertip, and it weighs $29 \mathrm{~g}$ for $75 \times 55 \times 30 \mathrm{~mm}$ dimensions. In Europe, GoTouchVR (France) developed a 1-DoF wearable device equipped with a mobile platform capable of applying pressure and making/breaking contact with the fingertip. It can exert up to $1.5 \mathrm{~N}$ on the skin, it weighs $40 \mathrm{~g}$ for $50 \times 12 \times 30 \mathrm{~mm}$ dimensions, it is wireless, and the battery guarantees up to 2 hours of playtime. These companies have also already been showing demonstrations of

*This research has received funding from the European Union's Horizon 2020 Research and Innovation Programme under Grant Agreement n ${ }^{\circ} 688857$ of the project "SoftPro."

†e-mail: spagnoletti@diism.unisi.it.

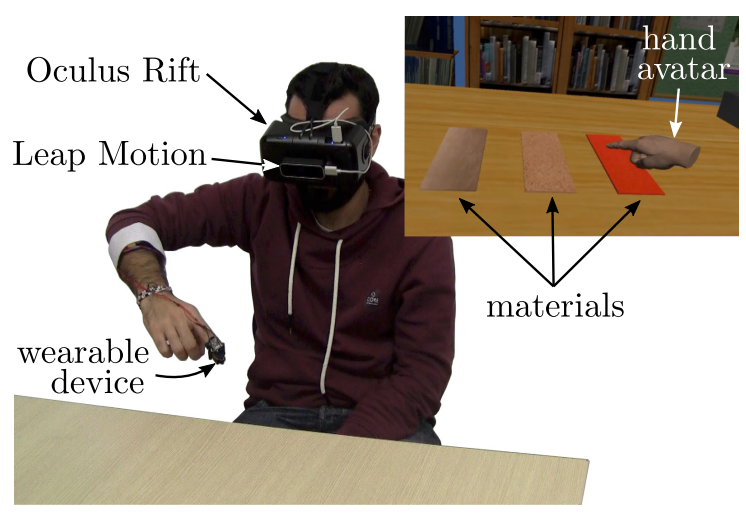

Figure 1: Wearable haptics experience for Virtual Reality. A Leap Motion tracks the motion of the fingertips, a hand avatar mimics the motion of the hand, and the fingertip wearable tactile device provides the user with pressure stimuli. The proposed device creates the sensation of making/breaking contact with virtual objects, and texture rendering. An Oculus Rift head-mounted display shows the virtual environment to the user.

their wearable haptics systems featuring immersive environments displayed through commercial virtual reality headsets [1,4]. For example, at CES 2017, both TACTAI and GoTouchVR showed their devices in a virtual reality application, using an Oculus Rift to render the scene and a Leap Motion controller for fingertip tracking. Also in the literature we can find several examples of wearable devices for the fingertips $[2,5,8,11]$. The challenge is to be able to provide effective stimuli while guaranteeing a high wearability, ergonomy, and low power consumption and cost. Pacchierotti et al. [10] have recently presented a review paper on wearable haptic devices for the fingertip and the hand, discussing also general guidelines for the development of such interfaces.

This work proposes a haptic-enabled immersive virtual reality system, shown in Fig. 1. A fingertip wearable device is able to recreate the sensations of touching objects made of different materials, providing pressure and texture rendering through a moving platform and a vibrotactile motor, respectively. Despite vibrations have been little considered in the past mostly because technological limitation of tiny actuators, the human sense of touch is capable of gathering valuable information through high-frequency vibrations about the features of an object one is in contact with [7]. Users are asked to interact with the VR environment and try to recognize the objects material by touching them. The VR environment is shown to the users through a head-mounted display (HMD).

\section{WeARABLE DEVICE DESCRIPTION}

We present a novel inexpensive wearable haptic device for cutaneous stimulation, developed following the design guidelines of [10]. It is shown in Fig. 2a, whereas a CAD sketch is shown in Fig. 2b. It 


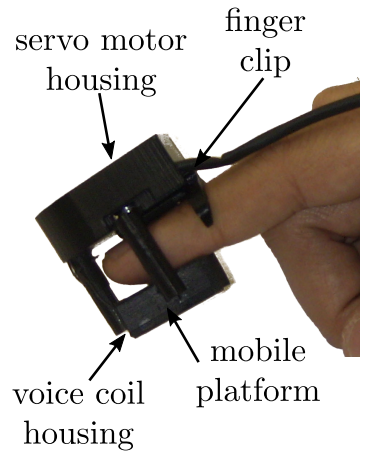

(a)

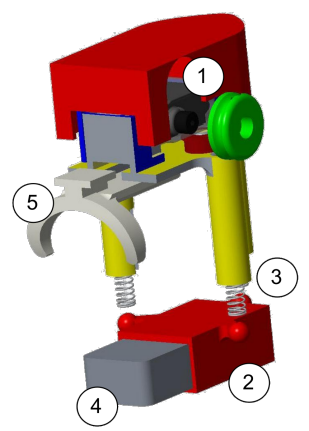

(b)
Figure 2: The wearable cutaneous device can provide the sensation of making/breaking contact with virtual objects, provide variable pressure, and render different textures.

is composed of a static upper body and a mobile end-effector. The upper body is located on the nail side of the finger, supporting one small servo motor (indicated with (1) in Fig. 2b), and the mobile end-effector is in contact with the finger pad (2). The two parts are connected by three cables (3) attached to the motor pulley, making the platform able to move towards or away from the fingertip. As a consequence, a variable pressure is provided directly to the user's fingertip to recreate the sensation of making and breaking contact with virtual objects. The end-effector platform houses a voice coil actuator (4) enabling the device to provide vibrotactile stimuli for texture rendering. Finally, a clip system (5) enables the user to easily fasten the device on the finger. The device is realized in Acrylonitrile Butadiene Styrene (ABS-Plus, Stratasys, USA) through the use of a commercial 3D printer. The servomotor is PWM-controlled and can provide a maximum torque of $0,8 \mathrm{~kg} \cdot \mathrm{cm}$. To render the texture of the virtual sufaces we used the The Penn Haptic Texture Toolkit (HaTT). The HaTT [3] includes 100 haptic texture and friction models. It was developed to provide haptics researchers with a method enabling the validation of their texture modeling and rendering methods. Thanks to this method, we were able to enrich our interaction with a compelling texture rendering, significantly improving the immersiveness of the user's experience.

The proposed device weighs $23 \mathrm{~g}$, the platform has a vertical displacement up to $20 \mathrm{~mm}$, and it can render a normal force up to $4 \mathrm{~N}$. Each device is connected to a wrist bracelet, connected to an external computer via bluetooth. The bracelet, capable of controlling up to 5 fingertip devices at a time, houses the controller and the audio amplifiers for the voice coil actuators. With respect to the wearable devices presented in [2], this device weighs less, is more compact, and can also render the textures of virtual objects and/or other vibrotactile stimuli related with specific events during the interaction (e.g., making/breaking the contact with the objects).

\section{HAPTIC-ENABLED VR EXPERIENCE}

We tested our wearable device in a Virtual Reality scenario. The experimental setup is shown in Fig. 1. The virtual environment is composed of a table placed in the middle of a furnished room. Three objects made of different materials are placed on the table: the red object is made of felt (the rightmost one in Fig. 1), the pale brown object is made of aluminum (the middle one in Fig. 1), and the dark brown object reproduces a brick surface (the leftmost one in Fig. 1). We chose to display these three representative materials, but the system is capable of rendering any of the 100 materials included in The Penn Haptic Texture Toolkit (HaTT) [3].

Ten participants (age $21-30$ ) wore an Oculus Rift and the wearable tactile device, as shown in Fig. 1. A Leap Motion controller was attached to the Oculus Rift to enable the tracking of the user's hand. A hand model was rendered in the virtual environment to mimic the user's hand pose. A mass-spring-damper model was then used to compute the interaction forces arising from the contact of the fingertip with the virtual environment. Our device were used to render these contact forces (through the algorithm presented in [9]), along with texture-related vibrotactile stimuli (through the algorithm presented in [3]), providing the user with the sensation of touching the objects in the scene. Users started by touching the three materials. After a familiarization trial of 2 minutes, subjects were presented with a different environment, having only one object on the table. The material of this single object was hidden (the object was shown in black), and users had to interact with it and guess its material (i.e., aluminum, brick, or felt).

At the end of the experiment, subjects rated their experience, evaluating the effectiveness of the provided feedback and the level of comfort of the device. The scores were given using a slider ranging from 0 (meaning "very low") to 10 (meaning "very high"). The feedback was rated 8.3 out of 10 , while the comfort of the system rated 7.9 out of 10 . Moreover, we evaluated the success rate in correctly recognizing the three materials. It was $63 \pm 15 \%$, which is significantly higher than the chance level of $33 \%$.

\section{REFERENCES}

[1] K. Bye. Tricking the brain is the only way to achieve a total haptics solution. https://web-beta.archive. org/web/20170121102102/http: //www . roadtovr. com/ tricking-brain-way-achieve-total-haptics-solution, 2017.

[2] F. Chinello, C. Pacchierotti, M. Malvezzi, and D. Prattichizzo. A three revolute-revolute-spherical wearable fingertip cutaneous device for stiffness rendering. IEEE Transactions on Haptics, PP(99):1-1, 2018. doi: 10.1109/TOH.2017.2755015

[3] H. Culbertson, J. J. L. Delgado, and K. J. Kuchenbecker. One hundred data-driven haptic texture models and open-source methods for rendering on $3 \mathrm{~d}$ objects. In IEEE Haptics Symposium (HAPTICS), pp. 319-325, 2014. doi: 10.1109/HAPTICS.2014.6775475

[4] J. Gaudiosi. Tactai gets in touch with ericsson for ar and vr "dynamic tactile wave" tech. https://uploadvr.com/ tactai-touch-ericsson/, 2017.

[5] B. T. Gleeson, S. K. Horschel, and W. R. Provancher. Design of a fingertip-mounted tactile display with tangential skin displacement feedback. IEEE Transactions on Haptics, 3(4):297-301, Oct 2010. doi: 10.1109/TOH.2010.8

[6] C. Grant. On eve of VR launches, hands-on with a new haptic ideal. https://web.archive.org/web/20160325214035/http: //www.polygon.com/virtual-reality/2016/3/25/11306368/ tactai-haptics-vr-virtual-reality-touch, 2016.

[7] R. L. Klatzky, S. J. Lederman, C. Hamilton, M. Grindley, and R. H. Swendsen. Feeling textures through a probe: Effects of probe and surface geometry and exploratory factors. Perception \& Psychophysics, 65(4):613-631, 2003. doi: 10.3758/BF03194587

[8] D. Leonardis, M. Solazzi, I. Bortone, and A. Frisoli. A wearable fingertip haptic device with 3 dof asymmetric 3-rsr kinematics. In IEEE World Haptics Conference (WHC), pp. 388-393, June 2015. doi: 10.1109/WHC.2015.7177743

[9] C. Pacchierotti, L. Meli, F. Chinello, M. Malvezzi, and D. Prattichizzo. Cutaneous haptic feedback to ensure the stability of robotic teleoperation systems. The International Journal of Robotics Research, 34(14):1773-1787, 2015. doi: 10.1177/0278364915603135

[10] C. Pacchierotti, S. Sinclair, M. Solazzi, A. Frisoli, V. Hayward, and D. Prattichizzo. Wearable haptic systems for the fingertip and the hand: Taxonomy, review, and perspectives. IEEE Transactions on Haptics, 10(4):580-600, 2017. doi: 10.1109/TOH.2017.2689006

[11] D. Prattichizzo, F. Chinello, C. Pacchierotti, and M. Malvezzi. Towards wearability in fingertip haptics: A 3-dof wearable device for cutaneous force feedback. IEEE Transactions on Haptics, 6(4):506-516, 2013. doi: $10.1109 / \mathrm{TOH} .2013 .53$ 